\title{
The importance of extent of choroid plexus cauterization in addition to endoscopic third ventriculostomy for infantile hydrocephalus: a retrospective North American observational study using propensity score-adjusted analysis
}

\author{
Aria Fallah, MD, MSc, FRCSC, ${ }^{1,2}$ Alexander G. Weil, MD, FRCSC, ${ }^{3}$ Kyle Juraschka, MD, ${ }^{4}$ \\ George M. Ibrahim, MD, PhD, ${ }^{4}$ Anthony C. Wang, MD, ${ }^{1}$ Louis Crevier, MD, MSc, FRCSC, ${ }^{3}$ \\ Chi-hong Tseng, PhD, ${ }^{5}$ Abhaya V. Kulkarni, MD, PhD, FRCSC, ${ }^{4}$ John Ragheb, MD, ${ }^{6}$ and \\ Sanjiv Bhatia, MD ${ }^{6}$
}

'Department of Neurosurgery, Mattel Children's Hospital, David Geffen School of Medicine at University of California, Los Angeles; ${ }^{2}$ Brain Research Institute, University of California, Los Angeles; ${ }^{5}$ Department of Medicine, David Geffen School of Medicine at University of California, Los Angeles, California; ' ${ }^{6}$ Department of Pediatric Neurosurgery, Nicklaus Children's Hospital, University of Miami, Florida; ${ }^{3}$ Division of Pediatric Neurosurgery, Department of Surgery, Sainte Justine Hospital, University of Montreal, Quebec; and "Division of Neurosurgery, The Hospital for Sick Children, University of Toronto, Ontario, Canada

OBJECTIVE Combined endoscopic third ventriculostomy (ETC) and choroid plexus cauterization (CPC) - ETV/CPC— is being investigated to increase the rate of shunt independence in infants with hydrocephalus. The degree of CPC necessary to achieve improved rates of shunt independence is currently unknown.

METHODS Using data from a single-center, retrospective, observational cohort study involving patients who underwent ETV/CPC for treatment of infantile hydrocephalus, comparative statistical analyses were performed to detect a difference in need for subsequent CSF diversion procedure in patients undergoing partial CPC (describes unilateral CPC or bilateral CPC that only extended from the foramen of Monro [FM] to the atrium on one side) or subtotal CPC (describes $\mathrm{CPC}$ extending from the FM to the posterior temporal horn bilaterally) using a rigid neuroendoscope. Propensity scores for extent of CPC were calculated using age and etiology. Propensity scores were used to perform 1) case-matching comparisons and 2) Cox multivariable regression, adjusting for propensity score in the unmatched cohort. Cox multivariable regression adjusting for age and etiology, but not propensity score was also performed as a third statistical technique.

RESULTS Eighty-four patients who underwent ETVICPC had sufficient data to be included in the analysis. Subtotal CPC was performed in 58 patients (69\%) and partial CPC in $26(31 \%)$. The ETV/CPC success rates at 6 and 12 months, respectively, were $49 \%$ and $41 \%$ for patients undergoing subtotal CPC and $35 \%$ and $31 \%$ for those undergoing partial CPC. Cox multivariate regression in a 48-patient cohort case-matched by propensity score demonstrated no added effect of increased extent of CPC on ETVICPC survival (HR 0.868, 95\% $\mathrm{Cl} 0.422-1.789, p=0.702$ ). Cox multivariate regression including all patients, with adjustment for propensity score, demonstrated no effect of extent of CPC on ETV/ CPC survival ( $\mathrm{HR} 0.845,95 \% \mathrm{Cl} 0.462-1.548, \mathrm{p}=0.586$ ). Cox multivariate regression including all patients, with adjustment for age and etiology, but not propensity score, demonstrated no effect of extent of CPC on ETVICPC survival (HR $0.908,95 \% \mathrm{Cl} 0.495-1.664, \mathrm{p}=0.755)$.

CONCLUSIONS Using multiple comparative statistical analyses, no difference in need for subsequent CSF diversion procedure was detected between patients in this cohort who underwent partial versus subtotal CPC. Further investigation regarding whether there is truly no difference between partial versus subtotal extent of CPC in larger patient populations and whether further gain in CPC success can be achieved with complete CPC is warranted.

https://thejns.org/doi/abs/10.3171/2017.7.PEDS16379

KEY WORDS choroid plexus cauterization; extent; outcome; time to event; hydrocephalus

ABBREVIATIONS $\mathrm{CCHU}=$ CURE Children's Hospital of Uganda; $\mathrm{CPC}=$ choroid plexus cauterization; $\mathrm{ETV}=$ endoscopic third ventriculostomy; ETVSS = ETV Success Score; FM = foramen of Monro; IVH = intraventricular hemorrhage.

SUBMITTED July 24, 2016. ACCEPTED July 7, 2017.

INCLUDE WHEN CITING Published online October 6, 2017; DOI: 10.3171/2017.7.PEDS16379. 
A FTER Walter Dandy first attempted choroid plexus cauterization (CPC) in 1918, several authors attempted CPC as a standalone treatment of infantile hydrocephalus with moderate success throughout the 20th century. ${ }^{6,12-15}$ Over the last decade, CPC has been reintroduced as an adjunct to endoscopic third ventriculostomy (ETV) for the treatment of pediatric hydrocephalus, particularly in sub-Saharan Africa, with the goal of improving shunt independence rates in children with risk factors for ETV failure, particularly infants or other patients with hydrocephalus related to myelomeningocele, postinfectious disease, or intraventricular hemorrhage of prematurity. ${ }^{19-22,24}$ This initial experience with combined ETV/ CPC in sub-Saharan Africa demonstrated significantly higher rates of shunt independence than ETV alone. ${ }^{19-22,24}$ Similarly, our group (J.R. and S.B.) has been performing ETV/CPC for hydrocephalus for over a decade in Haiti. ${ }^{25}$

Based on these findings, several groups have applied ETV/CPC to the treatment of infantile hydrocephalus in developed countries, including North American countries, France, and Japan, with acceptable safety and modest, albeit variable (38\%-57\%), success rates. ${ }^{2,10,11,16,26}$

\section{Rationale}

Controversy remains in areas of patient selection and nuances of surgical technique. One such nuance is the extent of CPC required to achieve surgical success, which has been operationally defined as decreased need for repeat CSF diversion procedures. ETV/CPC has been performed using both a flexible neuroendoscope and a rigid neuroendoscope. ${ }^{10,16,20,26}$ The use of a flexible neuroendoscope permits greater extent of CPC, as it provides access to choroid plexus beyond the posterior temporal horn and occasionally the anterior roof of the third ventricle, depending on the degree of hydrocephalus, ventricular morphology, and surgeon familiarity and experience with flexible neuroendoscopy techniques. The degree of CPC necessary to improve the success rate of ETV/CPC beyond that of ETV alone is currently unknown. Data from the use of ETV/CPC at the CURE Children's Hospital of Uganda (CCHU) have suggested that success rates improve with greater extent of CPC, with success rates for partial (unilateral) $\mathrm{CPC}$ and complete (bilateral) $\mathrm{CPC}$, respectively, showing doubling or quadrupling in comparison with the success rates seen ETV alone. ${ }^{24}$ These findings were used to develop and validate a scoring system, the CCHU ETV Success Score (ETVSS), that utilizes extent of CPC in the prediction model of ETV/CPC success in the sub-Saharan African population. ${ }^{24}$ It is uncertain whether these findings are externally valid and can be readily applied to developed countries in which the predominant etiology of infantile hydrocephalus is intraventricular hemorrhage of prematurity as opposed to postinfectious in nature. In a recent multicenter retrospective North American study of ETV/CPC for infantile hydrocephalus using both rigid and flexible neuroendoscopes, a nonsignificant trend toward improved outcome with more extensive (>90\%) CPC was observed, ${ }^{10}$ but there is a lack of evidence regarding the extent of CPC necessary to reduce the need for subsequent CSF diversion procedures in developed countries.
In the present study, we examine the extent of CPC (partial vs subtotal) using a propensity score-adjusted analysis in a previously published group of 84 children at a North American center who underwent ETV/CPC for infantile hydrocephalus to determine whether greater extent of CPC attained with a rigid neuroendoscope reduces the need for subsequent CSF diversion procedures.

\section{Methods}

\section{Protocol, Registration, and Reporting}

We developed a protocol before conducting this study but did not register it. We reported our findings in concordance with the STROBE (Strengthening the Reporting of Observational Studies in Epidemiology) guidelines. ${ }^{17}$

\section{Study Design and Patient Population}

We used previously collected data from a single-center, retrospective, observational cohort study ${ }^{26}$ to determine whether the extent of CPC affects surgical outcome in infants (age $<24$ months) with hydrocephalus being treated with combined ETV/CPC. Consecutive patients who had undergone an ETV and CPC with at least 1 postsurgical follow-up were included. During the period covered by the study, it was the surgical practice of the 2 senior authors (S.B. and J.R.) to perform an ETV and maximal cauterization of all visible and safely accessible choroid plexus using a rigid neuroendoscope as the primary treatment for hydrocephalus unless the surgery was not anatomically possible, access to the equipment or appropriate surgical team was limited, or there was parental preference for a CSF shunt.

\section{Surgical Technique}

The surgical technique for ETV/CPC used in this study has been described in detail previously.,26 The majority of the procedures were performed by 2 surgeons (S.B. and J.R.), with 2 other surgeons performing the remaining procedures. In brief, ETV was performed through a frontal bur hole, followed by ipsilateral cauterization of the choroid plexus. Septostomy and contralateral CPC were performed. Although the preoperative goal in every case was to completely cauterize any visible and accessible choroid plexus, the extent of CPC varied with degree of ventriculomegaly, septal anatomy, and the adequacy of visualization as the case progressed. Unilateral CPC was performed in cases in which the ventricular anatomy was aberrant (e.g., normal anatomical ependymal structures difficult to recognize) or when septostomy could not be performed. Prepontine scarring, when present intraoperatively, was removed to the extent deemed safely possible by the operating surgeon. A rigid neuroendoscope was used in all cases (Storz Oi [Karl Storz], Aesculap, Inc., or Claris [Medtronic]).

\section{Setting and Exposure of Interest}

All procedures were performed at Miami Children's Hospital or Jackson Memorial Hospital in Miami, Florida, between July 2007 and May 2014. Operative reports were retrospectively analyzed to determine the extent 
of CPC attained. To quantify and correlate the extent of CPC with outcome, the extent of subtotal CPC was subdivided into partial and subtotal. The extent was considered partial when CPC was unilateral or bilateral but only extended from the foramen of Monro (FM) to the atrium on one side. While we recognize that the complete choroid plexus (including such locations as within the fourth ventricle or at the roof of third ventricle) cannot be obtained by CPC regardless of the endoscope used, we classified extent of CPC as being subtotal when CPC extended from the FM to the posterior temporal horn bilaterally (Fig. 1). Other variables extracted included patient age at the time of surgery, etiology of hydrocephalus, and outcome data (length of follow-up or time to repeat CSF diversion procedure). ${ }^{26}$

\section{Outcome Measures}

The primary outcome measure in this study was defined as success or failure of the ETV/CPC procedure. Treatment failure was defined as the need for a repeated CSF diversion procedure (repeat ETV, CSF shunting, or reservoir requiring tapping) or death. The decision to perform a subsequent CSF diversion procedure was made at the discretion of the treating neurosurgeon. Although the rationale for these procedures was not recorded in this study, the decision to proceed with repeat CSF diversion was typically made when patients presented with signs and symptoms consistent with increased intracranial pressure and/or neurological or neurodevelopmental stagnation or decline, combined with ongoing or worsening ventriculomegaly or accelerated head growth.

\section{Description of Statistical Technique}

In the absence of clinical trials, clinical decision making is commonly informed by evidence from observational studies that are often biased with multiple confounding variables. This makes the attribution of causality for a desired outcome (CSF shunt freedom) to a specific clinical intervention (e.g., greater extent of ETV/CPC) challenging. Although a Cox proportional hazard analysis is an established and straightforward approach that can be used to build clinical prediction tools, several key assumptions must hold true (e.g., proportional hazard over time). In addition, the results may be biased and have low power if the event per variable ratio is less than 8:10. In contrast, propensity scores synthesize several confounding variables into a single measure. Propensity score analysis is suitable even when the event per variable ratio is less than 8:10. The appeal of this method is its ability to create a quasi-randomized comparison. Following the generation of the propensity score, it must be combined with other traditional multivariable methods such as matching, stratification, or regression for comparative analysis. ${ }^{5}$ The application of propensity score analysis to multiple treatment comparisons is not simple, and its interpretation can be challenging as propensity scores are often mistaken for risk scores. . $^{1,3,9}$

\section{Sample Size Calculation and Statistical Methods}

Using an $\alpha$ value of 0.05 , a $\beta$ value of 0.20 (given 26
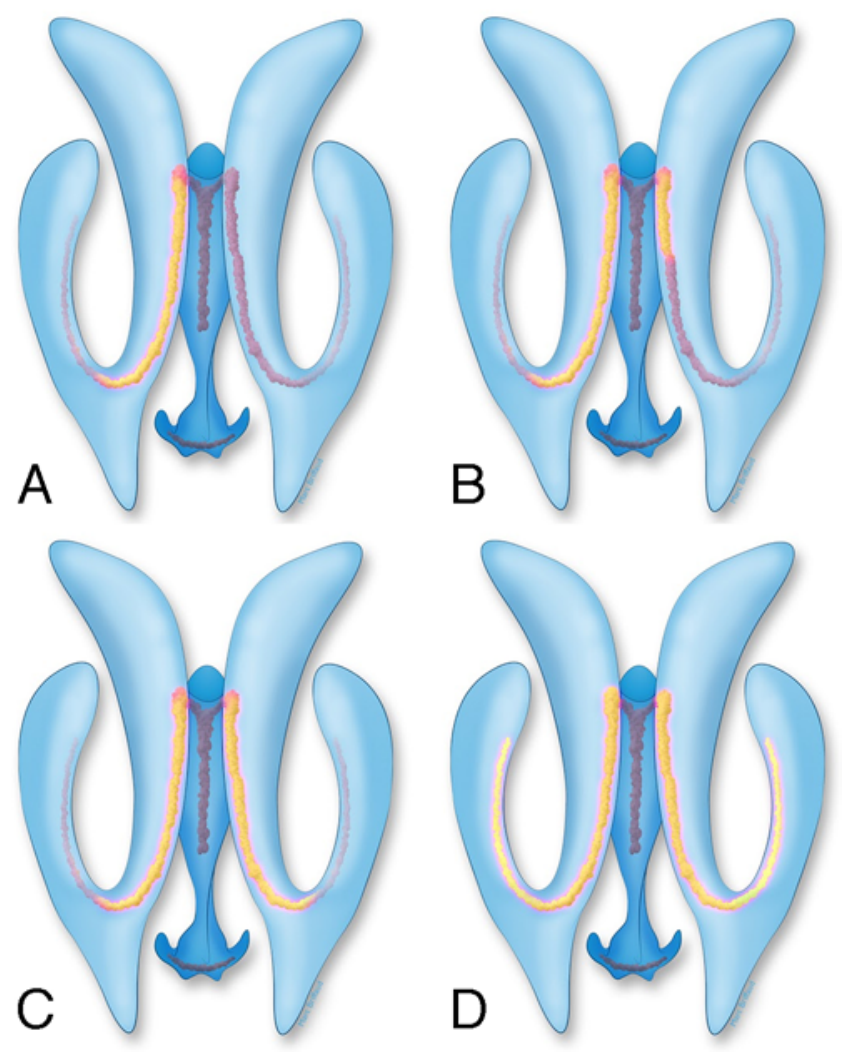

FIG. 1. Classification of extent of CPC. Partial CPC describes unilateral $\mathrm{CPC}(\mathrm{A})$ or bilateral CPC that only extended from the FM to the atrium on one side (B). Subtotal CPC describes CPC extending from the FM to the posterior temporal horn (atrium) bilaterally (C). Complete CPC (not included in this study) is performed from the FM to the anterior tip of the temporal horns bilaterally (D). Copyright Marc Briffaud. Published with permission. Figure is available in color online only.

participants in one cohort, and 58 participants in the other cohort), we had $73 \%$ power to detect a $30 \%$ absolute difference in the proportion of ETV/CPC success at 1 year's follow-up. For $80 \%$ power to detect a $10 \%$ difference in the proportion of success, we would require 376 participants per group (752 total).

The raw data are presented using descriptive statistics (means or medians, standard deviations, and ranges for continuous data; frequencies and percentages for categorical data). We used 3 statistical methods to investigate the effect of the extent of CPC on surgical outcome. We computed a propensity score for the extent of CPC for each participant, using age and hydrocephalus etiology. Variables for the propensity score were calculated using a multivariate logistic regression where subtotal versus partial CPC was a dichotomous treatment variable and the covariates were predictor variables (age and hydrocephalus etiology). The propensity scores represented the retrospective probability that a patient would receive partial versus subtotal CPC based on his or her age and hydrocephalus etiology. The propensity score was used to 1) case match using calipers 2 standard deviations of the logit of this variable and 2) perform a multivariate Cox regression adjusting for the propensity score and extent of CPC. Our third statistical technique was to perform a multivariate Cox regression 
adjusting for age and hydrocephalus etiology. Utilizing 3 separate techniques to examine this relationship enhances the statistical robustness of the analysis. To the extent that our results are similar, we can be confident in our conclusions. Hazard ratios, confidence intervals, and $\mathrm{p}$ values for these analyses are presented, reflecting the risk of treatment failure for subtotal CPC compared with partial CPC (control value). Kaplan-Meier curves were created to graph the overall time to repeat CSF diversion procedure. We accepted $p$ values of less than 0.05 as statistically significant. Statistical analyses were performed using IBM SPSS Statistics Version 22 (IBM Corp.) and R Statistical Software Version 3.2.3 (Foundation for Statistical Computing).

\section{Research Ethics Approval}

This study was approved by and compliant with the institutional review board at Miami Children's Hospital.

\section{Results \\ Participants}

From July 2007 to May 2014, 86 infants underwent ETV/CPC for hydrocephalus performed by 3 surgeons at Miami Children's Hospital or Jackson Memorial Hospital in Miami, Florida. One patient was lost to follow-up and excluded from the study. Another patient was excluded from the statistical analysis because of lack of operative data on extent of CPC performed. The etiology of hydrocephalus was intraventricular hemorrhage (IVH) of prematurity in 43 patients (51.2\%), myelomeningocele in 7 (8.3\%), congenital aqueductal stenosis in $12(14.3 \%)$, congenital communicating hydrocephalus in $6(7.1 \%)$, DandyWalker complex in $6(7.1 \%)$, postinfectious in $5(6.0 \%)$, and other cause in $5(6.0 \%)$. The mean age ( \pm standard deviation) at surgery was $4.3 \pm 3.8$ months (range 0-20 months). Subtotal CPC was performed in 58 patients $(69.0 \%)$ and partial CPC in 26 (31.0\%). ETV/CPC success rate was $49 \%$ and $41 \%$ for patients who underwent subtotal CPC and $35 \%$ and $31 \%$ for those who underwent partial CPC, at 6 and 12 months, respectively. Time-to-event analysis using Kaplan-Meier survival curves is depicted in Fig. 2. The median time to a repeat CSF diversion procedure was $3.0 \pm 1.2$ for the cohort that underwent partial and $7.0 \pm 3.4$ months for the cohort that underwent subtotal CPC (logrank [Mantel-Cox] test, $\mathrm{p}=0.837$ ).

\section{Effect of Extent of CPC on Outcome}

Statistical Technique 1: Case Matching by Propensity Score

Case matching using calipers 2 standard deviations of the logit of the propensity score resulted in an analysis of 48 participants (24 in each cohort). Comparative characteristics of the 2 cohorts are presented in Table 1. There was no statistically significant difference in patient age, hydrocephalus etiology, or the presence of prepontine scarring between the 2 cohorts. We plotted the distribution of the propensity scores before and after matching (Fig. 3). Following matching, the mean propensity scores (SD) of the cohorts that underwent subtotal CPC and partial CPC were $0.6786(0.0404)$ and $0.6793(0.0401)$, respectively ( $p$

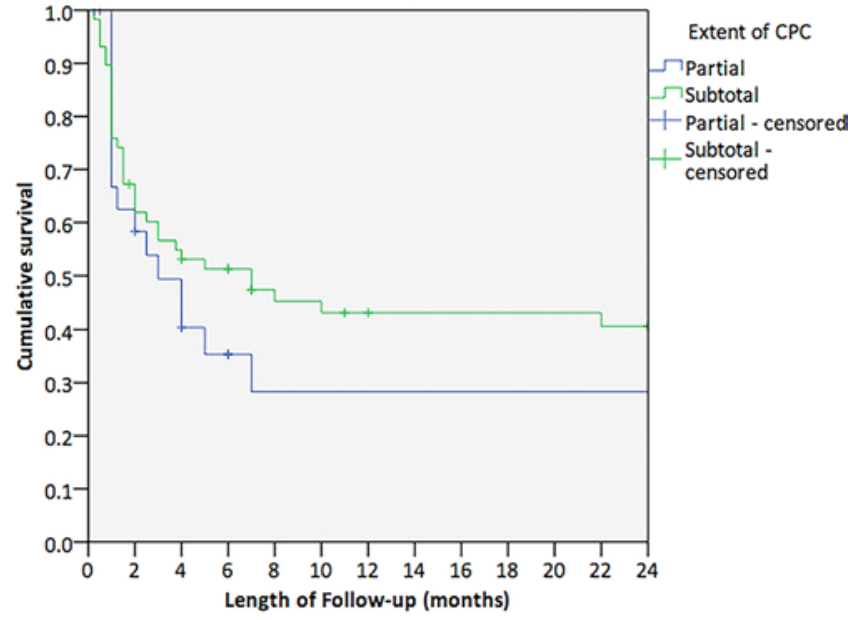

FIG. 2. ETVICPC survival function in all infantile hydrocephalus patients by extent of CPC. Figure is available in color online only.

$=0.952)$. Cox multivariate regression in this cohort adjusting for propensity score demonstrated no added effect from the extent of CPC on ETV/CPC survival (HR 0.868, 95\% CI 0.422-1.789, $\mathrm{p}=0.702)$. A Kaplan-Meier survival curve depicting this relationship is demonstrated in Fig. 4. The median time to repeat CSF diversion procedure was $3.0 \pm 1.1$ and $3.0 \pm 3.0$ months for the cohorts that underwent partial and subtotal CPC, respectively (log-rank [Mantel-Cox] test, $\mathrm{p}=0.674$ ).

Statistical Technique 2: Multivariate Cox Regression Adjusting for Propensity Score

Using a Cox multivariate regression on all participants, adjusting for the propensity score, we found that extent of CPC did not have an additional effect on ETV/CPC survival (HR 0.845, 95\% CI 0.462-1.548, $\mathrm{p}=0.586$ ).

\section{Statistical Technique 3: Multivariate Cox Regression}

Lastly, we performed a multivariate Cox regression adjusting for age and etiology, not utilizing the derived propensity score. The extent of CPC did not have an added effect on ETV/CPC survival (HR 0.908, 95\% CI 0.495$1.664, \mathrm{p}=0.755)$.

TABLE 1. Comparison of propensity score-matched cohorts

\begin{tabular}{lccc}
\hline \multicolumn{1}{c}{ Characteristic } & $\begin{array}{c}\text { Partial CPC } \\
\text { Cohort }\end{array}$ & $\begin{array}{c}\text { Subtotal } \\
\text { CPC Cohort }\end{array}$ & $\begin{array}{c}p \\
\text { Value }\end{array}$ \\
\hline Mean age in mos & 3.455 & 3.1413 & 0.727 \\
\hline Hydrocephalus etiology & & & 0.379 \\
\hline IVH of prematurity & $3(12.5)$ & $5(20.8)$ & \\
\hline Congenital AS & $13(54.1)$ & 9 & \\
\hline Myelomeningocele & $3(12.5)$ & 1 & \\
\hline Dandy-Walker malformation & $5(20.8)$ & 9 & \\
\hline Intraoperative prepontine scarring & & & 0.617 \\
\hline No & $15(62.5)$ & $18(33.3)$ & \\
\hline Yes & $8(33.3)$ & $5(20.8)$ & \\
\hline
\end{tabular}

$\mathrm{AS}=$ aqueductal stenosis.

Data are presented as number of patients (\%) unless otherwise indicated. 


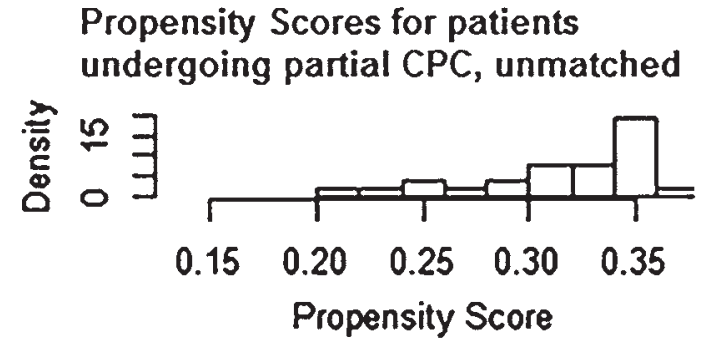

\section{Propensity Scores for patients undergoing subtotal $\mathrm{CPC}$, unmatched}

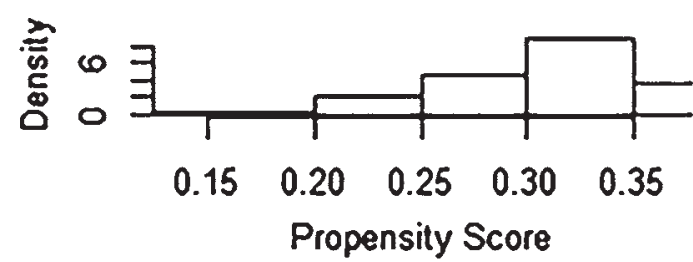

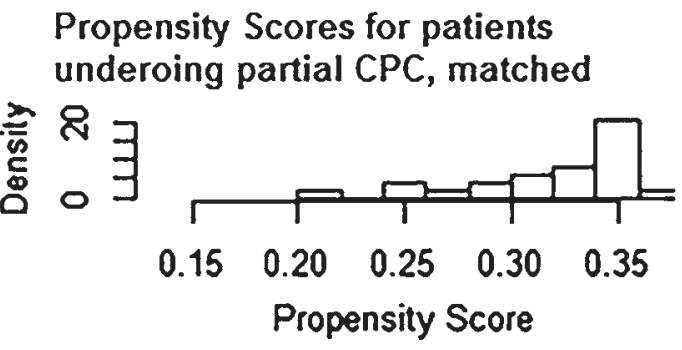

\section{Propensity Scores for patients undergoing subtotal $\mathrm{CPC}$, matched}

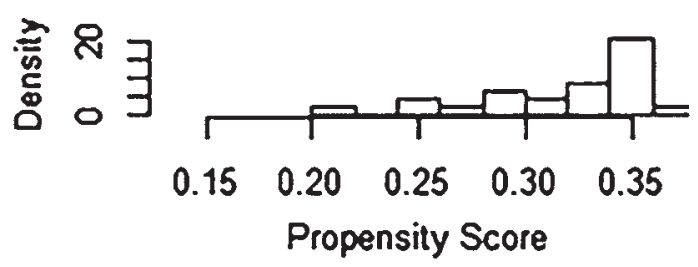

FIG. 3. Histogram demonstrating the distribution of propensity scores before and after matching among participants that underwent partial (upper) and subtotal (lower) CPC.

\section{Discussion}

\section{Role of Extent of CPC in Infantile Hydrocephalus}

In this paper, we report the results of analysis of the impact of extent of CPC on efficacy following ETV/CPC with a rigid neuroendoscope, based on data from a singlecenter North American retrospective observational cohort study. ${ }^{26}$ Using multiple statistical techniques, we did not find a statistically significant difference that supported subtotal versus partial CPC to reduce the need for subsequent CSF diversion in this cohort.

Although several studies have suggested that the addition of CPC improves outcome compared with that expected from ETV alone, the role of CPC and extent of CPC have been topics of debate..$^{2,4,10,16,20,23,26}$ Because flexible neuroendoscopes allow entry into the temporal horn and more complete CPC, the debate on extent of CPC parallels the debate on whether there is any difference in efficacy with the use of a rigid or flexible neuroendoscope. The findings in our study suggest that it is still inconclusive whether subtotal CPC improves efficacy over partial $\mathrm{CPC}$ when using a rigid neuroendoscope to treat primarily posthemorrhagic hydrocephalus. The goal of CPC is to provide alteration in CSF dynamics (either by decreasing CSF output sufficiently to reestablish a balance of CSF output and resorption or by reducing CSF pulsation amplitudes).$^{8}$ However, the amount of CPC required to establish a normal equilibrium in any given patient is currently unknown.

The findings in our study contrast with those of some of the prior studies that have examined the relationship between extent of CPC and need for subsequent CSF diversion, particularly extensive research in sub-Saharan Africa. ${ }^{16,20-22,24}$ In the sub-Saharan experience with combined ETV/CPC using a flexible neuroendoscope, extent of CPC was identified as significant predictor of outcome in a multivariable logistic regression model, and extent of CPC is one of the variables used in the development of the CCHU
ETVSS. ${ }^{24}$ Although there is some indirect evidence in North America that use of a flexible neuroendoscope (suggesting more extensive CPC) improves success, the data are limited and contradictory. $4,10,16,26$ In a study of 91 consecutive North American patients undergoing ETV/CPC with a flexible neuroendoscope, Stone et al. demonstrated a success rate of $57 \%$ at 12 months, exceeding both the predicted ETVSS (45\%), and success rates documented in other North American studies (37\%-50\%), which often used exclusively rigid neuroendoscopes or both flexible and rigid neuroendoscopes.,10,16,26 The increased success rate achieved may potentially be explained by increased extent of CPC obtained using a flexible neuroendoscope. A recent multicenter North American study involving 36 infants treated with ETV/CPC adds some weight to this possibility, as it showed a trend toward improved outcome when $>90 \%$ extent of lateral ventricle CPC was achieved

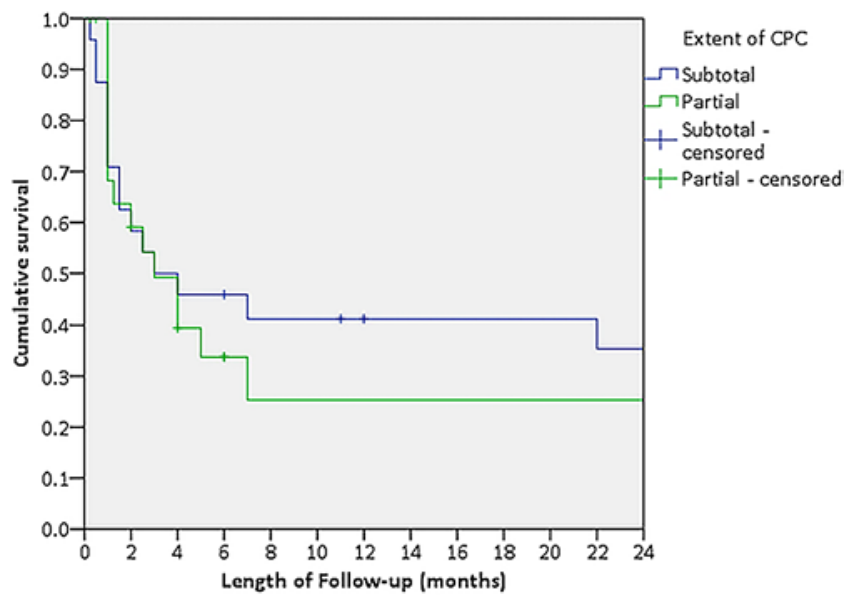

FIG. 4. ETV/CPC survival function in propensity score-matched sample of infantile hydrocephalus patients by extent of CPC. Figure is available in color online only. 
TABLE 2. Study design considerations for a trial to determine the effect of extent of CPC in ETV/CPC for the treatment of infantile hydrocephalus

\begin{tabular}{|c|c|}
\hline Consideration & Description \\
\hline Population & $\begin{array}{l}\text { The study should be restricted to consecutive infants younger than } 2 \text { years with untreated hydrocephalus of any etiology who would } \\
\text { be eligible for ETVICPC. } \\
\text { Inclusion criteria: 1) symptomatic hydrocephalus requiring treatment, 2) no previous definitive treatment for hydrocephalus, 3) age } \\
<24 \text { months at time of surgery, 4) preoperative MRI that confirms the diagnosis, and 5) ability to participate in follow-up for at } \\
\text { least } 5 \text { years. } \\
\text { Exclusion criteria: 1) intracranial tumor, 2) gestational age <37 weeks at time of surgery, 3) untreated spina bifida, and 4) severe } \\
\text { dysmorphic features. }\end{array}$ \\
\hline $\begin{array}{l}\text { Other design } \\
\text { considerations }\end{array}$ & $\begin{array}{l}\text { To improve external validity, the included patients should comprise a multicenter and international cohort encompassing a large } \\
\text { range of socioeconomic backgrounds and diversity in the etiology of hydrocephalus. } \\
\text { Follow-up should be through clinical visits that include palpation of the anterior fontanelle, head circumference measurements, and } \\
\text { annual MRI examinations to assess changes in ventricular size. }\end{array}$ \\
\hline Bias & $\begin{array}{l}\text { An inherent bias for this procedure is the degree of CPP that a surgeon can perform safely. Because experience and training is } \\
\text { important, we recommend an expertise-based trial design in which surgeons with at least } 10 \text { previous successful ETV/CPC } \\
\text { procedures perform this procedure at their center.* }\end{array}$ \\
\hline Intervention & Rigid or flexible neuroendoscopy with the preoperative goal of ETV and maximum safe CPP. \\
\hline $\begin{array}{l}\text { Different levels } \\
\text { of exposure of } \\
\text { interest }\end{array}$ & $\begin{array}{l}\text { Postoperative MRI with contrast and quantification of the degree of CPP and the assessment of flow void at the level of the tuber } \\
\text { cinereum. Using software for volumetric analysis, the radiologist will assign a percentage of total choroid plexus cauterized in } \\
\text { the lateral ventricles. This number should be computed independently and in duplicate by } 2 \text { radiologists and an average value } \\
\text { calculated. }\end{array}$ \\
\hline Sample size & $\begin{array}{l}\text { To detect a } 10 \% \text { difference in outcome (need for repeat CSF diversion procedure), a total of } 752 \text { participants ( } 376 \text { per group) would } \\
\text { be required to achieve } 80 \% \text { power. }\end{array}$ \\
\hline $\begin{array}{l}\text { Outcome mea- } \\
\text { sures }\end{array}$ & $\begin{array}{l}\text { Well-defined objective criteria (persistent enlargement of ventricles or crossing of head circumference growth curves) for repeat } \\
\text { CSF diversion intervention; health status outcome at } 1 \text { and } 5 \text { years following surgery. }\end{array}$ \\
\hline Blinding & Blinding of outcome assessors (assessment of need for subsequent CSF diversion procedures by an independent neurosurgeon). \\
\hline Follow-up & $\begin{array}{l}\text { Loss-to-follow-up should be minimized to }<10 \% \text { at } 1 \text { year. Given the anticipated loss-to-follow-up, the sample size should be in- } \\
\text { creased accordingly to keep the study well powered. Time-to-event analyses will allow for censoring of patients lost to follow-up. }\end{array}$ \\
\hline $\begin{array}{l}\text { Statistical } \\
\text { analyses }\end{array}$ & $\begin{array}{l}\text { Multivariable Cox regression analysis for time to repeat CSF diversion procedure, determining whether the percentage of choroid } \\
\text { plexus cauterized after controlling for potential confounding variables (age of the participant at the time of surgery and etiology } \\
\text { of hydrocephalus) is statistically significant. }\end{array}$ \\
\hline
\end{tabular}

* In accordance with the recommendations of Devereaux et al. ${ }^{7}$

(82\% vs $36 \%$, with ETVSS of $51 \%$ and $50 \%$, respectively, $\mathrm{p}=0.0501) .{ }^{10}$ However, the cohort in which this trend was identified was relatively small, and multiple confounders, including surgeon's learning curve (flexible endoscopes were used later in the series) and potential for uncontrolled differences in patients receiving $>90 \% \mathrm{CPC}$, limit the interpretation of the results. A recent study by our research group compared flexible to rigid endoscopy for ETV/CPC and demonstrated that the group that underwent ETV/CPC via flexible endoscopy had a higher frequency of complete CPC (98.9\% vs $86.0 \%)$, but, as in this study, after propensity score-matched analysis, no difference in outcome between the groups was found despite differences in extent of CPC completed. ${ }^{18}$

Significant differences in patient populations (particularly differing age and hydrocephalus etiology), selection biases, outcome definitions, and surgical technique exist between the cohorts presented in the current literature. Our study presents a cohort in which patients were selected for ETV/CPC (instead of ETV alone or a shunting procedure) without set criteria. In general, patients were selected for ETV/CPC who were considered to be at higher risk for ETV failure. Thus, the presented data may reflect a subset of patients with a high likelihood of ETV failure (as reflected in low median ETVSS previously reported for this cohort of 40\%). To our knowledge, no data currently exist on whether CPC results in a greater benefit in patients with lower or higher predicted ETV success rates. Additionally, the decision to proceed with a subsequent CSF diversion procedure varies among surgeons, and the fact that some degree of ventriculomegaly typically persists in these patients introduces a significant source of bias into the body of evidence currently available, which consists of modestly sized cohorts $\left(<100\right.$ patients). ${ }^{2,10,16,26}$ Furthermore, other studies in similar patient populations, such as patients with IVH of prematurity, have had similar efficacy (40\%) using flexible and rigid endoscopes. . $^{4,23,26}$

A further limitation of all of these studies is the nonobjective assessment of extent of CPC. Studies vary as to whether these data were collected prospectively or retrospectively. Nonetheless, while surgeon estimates of extent of CPC likely correlate with actual extent of CPC attained, it is an inherently blunt and perhaps inaccurate measure. A radiological surrogate of extent of CPC might better demonstrate any true relationship with outcome. As well, the current study used a dichotomized categorization of ex- 
tent of CPC based on attempts at unilateral versus bilateral $\mathrm{CPC}$, without objective data to suggest there is significant difference in overall CSF production or pulsatility between these 2 categories. Thus, the lack of significant difference in clinical outcome may be explained by an insignificant difference in physiological sequelae of these degrees of CPC. It is also likely that the degree of CPC necessary to achieve physiological balance varies between patients, thus making a true difference even more difficult to detect.

\section{Limitations}

This study is limited by multiple factors, including small patient sample size limiting statistical power, retrospective nature of the data collection, nonblinded data collection, and nonstandardized criteria for patient selection for the ETV/CPC procedure, introducing a selection bias. In particular, extent of CPC was determined through review of surgical notes. This introduces observer bias as well as entailing inherent inaccuracy in data related to intraoperative assessment of extent of CPC, as discussed previously. The division of this cohort into 2 discrete groups is also limited by the fact that although the extent of CPC was very different in the 2 groups, there is a possibility that a small subset of patients in each group had similar extents of CPC. The extent of CPC in a patient on the extensive side of the partial CPC category may, in theory, closely resemble the extent of a patient on the less extensive side of the subtotal CPC category. Development of a blinded, objective measure of extent of CPC, such as analysis based on pre- versus postoperative MRI, may be a useful tool for future analyses of extent of CPC on outcome. The single-center nature of this study may limit external validity of the findings. However, current published data are limited to single-center cohort, or small, multicenter cohort studies.

The primary limitation of this study is the limited statistical power to detect a difference in outcome. Even in the most convincing North American data suggesting benefit of the addition of CPC to ETV, the absolute risk reduction of treatment failure for ETV/CPC compared with ETVSS (i.e., ETV alone) is $14 \% .{ }^{16}$ Intuitively, any difference in outcome between varying degrees of CPC would, therefore, be expected to be less than $14 \%$. As mentioned previously, our analysis would have required 376 participants per group (752 total) in order to attain $80 \%$ power to detect a $10 \%$ difference in outcome. The rarity of ETV/ $\mathrm{CPC}$ in infantile hydrocephalus in North America is a major barrier to attaining sufficient sample size to adequately address this issue with adequate statistical power. A multicenter study powered to further explore this question is being planned. Recommendations for an ideal study design that would best address effect of extent of CPC on outcome are presented in Table 2.

\section{Conclusions}

Extent of CPC (partial vs subtotal) performed with a rigid neuroendoscope did not demonstrate a statistically significant difference in rates of treatment failure in this single-center North American cohort. Further studies with larger patient populations, analyses of the relationship of extent of CPC to treatment success in specific etiologies, and use of objective measures of extent of CPC may help to define the role of ETV/CPC, and specifically the extent of CPC required, in the treatment of infantile hydrocephalus in developed countries.

\section{Acknowledgments}

We would like to thank Marc Briffaud for creating the illustration of the extent of CPC (Fig. 1).

\section{References}

1. Adams H, Adams HH, Jackson C, Rincon-Torroella J, Jallo GI, Quiñones-Hinojosa A: Evaluating extent of resection in pediatric glioblastoma: a multiple propensity score-adjusted population-based analysis. Childs Nerv Syst 32:493-503, 2016

2. Beuriat PA, Szathmari A, Grassiot B, Plaisant F, Rousselle C, Mottolese C: Role of endoscopic third ventriculostomy in the management of myelomeningocele-related hydrocephalus: a retrospective study in a single French institution. World Neurosurg 87:484-493, 2016

3. Biondi-Zoccai G, Romagnoli E, Agostoni P, Capodanno D, Castagno D, D'Ascenzo F, et al: Are propensity scores really superior to standard multivariable analysis? Contemp Clin Trials 32:731-740, 2011

4. Chamiraju P, Bhatia S, Sandberg DI, Ragheb J: Endoscopic third ventriculostomy and choroid plexus cauterization in posthemorrhagic hydrocephalus of prematurity. J Neurosurg Pediatr 13:433-439, 2014

5. D'Agostino RB Jr: Propensity score methods for bias reduction in the comparison of a treatment to a non-randomized control group. Stat Med 17:2265-2281, 1998

6. Dandy WE: Extirpation of the choroid plexus of the lateral ventricles in communicating hydrocephalus. Ann Surg 68:569-579, 1918

7. Devereaux PJ, Bhandari M, Clarke M, Montori VM, Cook DJ, Yusuf S, et al: Need for expertise based randomised controlled trials. BMJ 330:88, 2005

8. Kahle KT, Kulkarni AV, Limbrick DD Jr, Warf BC: Hydrocephalus in children. Lancet 387:788-799, 2016

9. Kulkarni AV, Drake JM, Kestle JR, Mallucci CL, Sgouros S, Constantini S: Endoscopic third ventriculostomy vs cerebrospinal fluid shunt in the treatment of hydrocephalus in children: a propensity score-adjusted analysis. Neurosurgery 67:588-593, 2010

10. Kulkarni AV, Riva-Cambrin J, Browd SR, Drake JM, Holubkov R, Kestle JR, et al: Endoscopic third ventriculostomy and choroid plexus cauterization in infants with hydrocephalus: a retrospective Hydrocephalus Clinical Research Network study. J Neurosurg Pediatr 14:224-229, 2014

11. Ogiwara H, Uematsu K, Morota N: Obliteration of the choroid plexus after endoscopic coagulation. J Neurosurg Pediatr 14:230-233, 2014

12. Pople IK, Edwards RJ, Aquilina K: Endoscopic methods of hydrocephalus treatment. Neurosurg Clin N Am 12:719735, viii, 2001

13. Pople IK, Ettles D: The role of endoscopic choroid plexus coagulation in the management of hydrocephalus. Neurosurgery 36:698-702, 1995

14. Pople IK, Griffith HB: Control of hydrocephalus by endoscopic choroid plexus coagulation-long-term results and complications. Eur J Pediatr Surg 3 (Suppl 1):17-18, 1993

15. Putnam TJ: Surgical treatment of infantile hydrocephalus. Calif Med 78:29-32, 1953

16. Stone SS, Warf BC: Combined endoscopic third ventriculostomy and choroid plexus cauterization as primary treatment for infant hydrocephalus: a prospective North American series. J Neurosurg Pediatr 14:439-446, 2014 
17. von Elm E, Altman DG, Egger M, Pocock SJ, Gøtzsche PC, Vandenbroucke JP: The Strengthening the Reporting of Observational Studies in Epidemiology (STROBE) statement: guidelines for reporting observational studies. Epidemiology 18:800-804, 2007

18. Wang S, Stone S, Weil AG, Fallah A, Warf BC, Ragheb J, et al: Comparative effectiveness of flexible versus rigid neuroendoscopy for endoscopic third ventriculostomy and choroid plexus cauterization: a propensity score-matched cohort and survival analysis. J Neurosurg Pediatr 19:585-591, 2017

19. Warf B, Ondoma S, Kulkarni A, Donnelly R, Ampeire M, Akona J, et al: Neurocognitive outcome and ventricular volume in children with myelomeningocele treated for hydrocephalus in Uganda. J Neurosurg Pediatr 4:564-570, 2009

20. Warf BC: Comparison of endoscopic third ventriculostomy alone and combined with choroid plexus cauterization in infants younger than 1 year of age: a prospective study in 550 African children. J Neurosurg 103 (6 Suppl):475-481, 2005

21. Warf BC: Endoscopic third ventriculostomy and choroid plexus cauterization for pediatric hydrocephalus. Clin Neurosurg 54:78-82, 2007

22. Warf BC, Campbell JW: Combined endoscopic third ventriculostomy and choroid plexus cauterization as primary treatment of hydrocephalus for infants with myelomeningocele: long-term results of a prospective intent-to-treat study in 115 East African infants. J Neurosurg Pediatr 2:310-316, 2008

23. Warf BC, Campbell JW, Riddle E: Initial experience with combined endoscopic third ventriculostomy and choroid plexus cauterization for post-hemorrhagic hydrocephalus of prematurity: the importance of prepontine cistern status and the predictive value of FIESTA MRI imaging. Childs Nerv Syst 27:1063-1071, 2011

24. Warf BC, Mugamba J, Kulkarni AV: Endoscopic third ven- triculostomy in the treatment of childhood hydrocephalus in Uganda: report of a scoring system that predicts success. $\mathbf{J}$ Neurosurg Pediatr 5:143-148, 2010

25. Weil AG, Fallah A, Bhatia S, Ragheb J: Pediatric neurosurgery in the developing world: the Haiti experience. J Craniofac Surg 26:1061-1065, 2015

26. Weil AG, Fallah A, Chamiraju P, Ragheb J, Bhatia S: Endoscopic third ventriculostomy and choroid plexus cauterization with a rigid neuroendoscope in infants with hydrocephalus. J Neurosurg Pediatr 17:163-173, 2016

\section{Disclosures}

The authors report no conflict of interest concerning the materials or methods used in this study or the findings specified in this paper.

\section{Author Contributions}

Conception and design: Fallah, Weil. Acquisition of data: Weil. Analysis and interpretation of data: Fallah, Weil. Drafting the article: Fallah, Weil, Juraschka. Critically revising the article: Fallah, Weil, Ibrahim, Wang, Crevier, Kulkarni, Ragheb, Bhatia. Reviewed submitted version of manuscript: all authors. Approved the final version of the manuscript on behalf of all authors: Falla. Statistical analysis: Fallah, Ibrahim, Tseng. Study supervision: Fallah, Weil.

\section{Correspondence}

Aria Fallah, Department of Neurosurgery, David Geffen School of Medicine at UCLA, UCLA Mattel Children's Hospital, 300 Stein Plaza, Ste. 525, Los Angeles, CA 90095-6901. email: afallah@ mednet.ucla.edu. 